\title{
A bacterial artificial chromosome transgenic mouse model for visualization of neurite growth
}

\author{
TAO Tao, CHEN Chen, SUN Jie, PENG YaJing \& ZHU MinSheng* \\ Model Animal Research Center and MOE Key Laboratory of Model Animal for Disease Study, Nanjing University, Nanjing 210061, China
}

Received July 9, 2014; accepted September 29, 2014

\begin{abstract}
Class III $\beta$-tubulin (Tubb3) is a component of the microtubules in neurons and contributes to microtubule dynamics that are required for axon outgrowth and guidance during neuronal development. We here report a novel bacterial artificial chromosome (BAC) transgenic mouse line that expresses Class III $\beta$-tubulin fused to mCherry, an improved monomeric red fluorescent protein, for the visualization of microtubules during neuronal development. A BAC containing Tubb3 gene was modified by insertion of mCherry complementary DNA downstream of Tubb3 coding sequence via homologous recombination. mCherry fusion protein was expressed in the nervous system and testis of the transgenic animal, and the fluorescent signal was observed in the neurons that located in the olfactory bulb, cerebral cortex, hippocampal formation, cerebellum, as well as the retina. Besides, Tubb3-mCherry fusion protein mainly distributed in neurites and colocalized with endogenous Class III $\beta$-tubulin. The fusion protein labels Purkinje cell dendrites during cerebellar circuit formation. Therefore, this transgenic line might be a novel tool for scientific community to study neuronal development both in vitro and in vivo.
\end{abstract}

Tubb3, mCherry, BAC transgenic mouse, neuronal development, Purkinje cells

Citation: Tao T, Chen C, Sun J, Peng YJ, Zhu MS. A bacterial artificial chromosome transgenic mouse model for visualization of neurite growth. Sci China Life Sci, 2015, 58: 373-378, doi: 10.1007/s11427-015-4820-9

Coordination of actin filament and microtubules in the axonal growth cone is required for proper axon growth and guidance, which are key processes during neuronal development [1]. In the past decades, visualizing the cytoskeleton in the growth cone is mostly achieved from antibodyassociated immunofluorescence of fixed neurons or in live neurons by transfection of fluorescent proteins tagged cytoskeletal proteins or their binding proteins. Labeling the cytoskeletal components in live cells had been used since long time ago [2] and already shed some light for neuroscientists to study the behavior of actin filament or microtubules in developing neurons $[3,4]$, but there actually lack many genetic tools so far that could express fluorescent proteins to label cytoskeleton without in vitro transfection or infection by viruses. Due to the low efficiency of transfection or viral

*Corresponding author (email: zhums@nju.edu.cn) infection in primary neurons, it is necessary to establish a transgenic line with specific expression of cytoskeleton proteins tracked by fluorescent protein. There are already available transgenic lines termed Lifeact mice which take the advantage of fluorescent proteins-conjugated a 17-amino-acid Lifeact peptide to visualize F-actin in primary cultured live cells and whole animals [5], but unfortunately and to our knowledge, there was no line available for visualizing neuronal microtubules.

We thus intend to generate a novel transgenic mouse line that expresses fluorescent protein fused with protein to label the microtubules in developing neurites. Microtubules are tubular structures consisting of 13 protofilaments, which are dynamically assembled from linear arrayed $\alpha$ - and $\beta$ tubulins dimers. Both $\alpha$ - and $\beta$-tubulin isotypes are encoded by multigene families, and at least six functional genes for $\alpha$ - and seven for $\beta$-tubulins had been identified in verte- 
brates [6]. We chose Class III $\beta$-tubulin (Tubb3) that is expressed only in testes sertoli cells and differentiated neurons [7], and is extensively used as differentiated neuronal marker. Labeling neuronal specific $\beta$-tubulin in the brain makes it possible to distinguish neurons from glia cells, thereby study neuronal rather than glial outgrowth and guidance in cell culture system and in vivo. In addition, human genetics study had demonstrated the required role of $T u b b 3$ for proper axon guidance [8]. To overcome the positional effect of conventional transgene approach, we employed bacterial artificial chromosome (BAC) based recombineering technology to generate a transgenic mouse line that expresses Class III $\beta$-tubulin fused to mCherry, an improved monomeric red fluorescent protein, to illuminate microtubules in the neurons. This new transgenic line is expected to be a useful tool to study microtubules dynamics during development.

\section{Materials and methods}

\subsection{Generation of transgenic mouse}

The 129s7/AB2.2 BAC Clone bMQ_125j13 containing the $T u b b 3$ gene was ordered from the Wellcome Trust Sanger Institute and modified by homologous recombination in E. coli using the Counter-Selection BAC Modification Kit (Gene Bridges, Germany). The E. coli strain DH10B carrying the BAC was firstly transformed by pRedET, which would express genes mediating Red/ET recombination by addition of L-arabinose and a temperature shift from 30 to $37^{\circ} \mathrm{C}$. A pair of primers that contain 60 -bp homologous arms at $5^{\prime}$-terminal was prepared and used to amplify the rpsL-neo cassette by polymerase chain reaction (PCR). The PCR product was gel-purified and electroporated into DH10B competent cells carrying the BAC and expressing Red/ET genes for homologous recombination. Recombinated BAC clone was selected by Kanamycin resistance, and verified by PCR determination. pRedET further transformed the cells to maintain the expression of pRed/ET genes for next step of recombination. Similar to the previous step, another pair of primers that contain the same 60-bp homologous arms and 15-bp sequence encoding Gly-Gly-Ser-Gly-Gly linker in the forward primer was prepared and used to amplify mCherry cDNA from pSAT6-mCherry-C1B (pE3275). The PCR product was then gel-purified and electroporated into competent $\mathrm{DH} 10 \mathrm{~B}$ host strain carrying rpsL-neo modified BAC for homologous recombination. Positive colony was counter-selected by Streptomycin insensitivity and PCR screening, and then verified by sequencing using primers located flanking the insertion site. The final correctly modified BAC was harvested from $500 \mathrm{~mL}$ overnight culture and purified using the NucleoBond BAC100 kit (Macherey-Nagel, Germany), and diluted in the microinjection buffer $\left(10 \mathrm{mmol} \mathrm{L}^{-1}\right.$ Tris-HCl, $0.1 \mathrm{mmol} \mathrm{L}^{-1}$ EDTA, $100 \mathrm{mmol} \mathrm{L}{ }^{-1} \mathrm{NaCl}, 30$ nmol L ${ }^{-1}$ Spermin and $70 \mathrm{nmol} \mathrm{L}{ }^{-1}$ Spermidine). After restriction analysis to determine the integrity of the modified BAC, the DNA was microinjected to C57BL/6 pronuclear to generate founder mice, which were selected by genotyping using primers: forward 5'-CTTCGCCTGGGACATCCTGT-3' and reverse 5'-TTGACCTCAGCGTCGTAGTG-3'. All procedures involving animals were carried out following the protocols approved by the Institutional Animal Care and Use Committee of Model Animal Research Center.

\subsection{Quantitative real-time PCR}

Mouse livers were incubated in lysis buffer $\left(100 \mathrm{mmol} \mathrm{L}^{-1}\right.$ Tris-HCl, $\mathrm{pH} 8.8 ; 50 \mathrm{mmol} \mathrm{L}^{-1}$ EDTA; $0.5 \%$ SDS; 200 mmol L ${ }^{-1} \mathrm{NaCl}$; $0.2 \mathrm{mg} \mathrm{mL}^{-1}$ Protainase $\mathrm{K}$ ) at $60^{\circ} \mathrm{C}$ overnight. Genomic DNA was further extracted by phenol/chloroform, precipitated by EtOH, and dissolved in TE buffer. $10 \mathrm{ng} \mu^{-1}$ genomic DNA was used as PCR template, using primers for Tubb3: forward 5'-AGGTGCGTGAGGAGTACCC-3' and reverse 5'-AGGGCTTCATTGTCGATGCAG-3', primers for Actb as loading control: forward 5'CCAACTGGGACGACATGGAG-3' and reverse 5'-TACCCGGGATACTGACCTGG-3'.

\subsection{Western blot}

Mice were sacrificed by cervical dislocation and tissues were isolated and lysised in modified radioimmunoprecipitation assay (RIPA) buffer, supplemented with $1 \times$ Cocktail proteinase inhibitor (Complete, Roche Molecular Biochemicals, USA) and $1 \mathrm{mmol} \mathrm{L}^{-1}$ phenylmethanesulfonylfluoride (PMSF). Equal amounts of protein lysates of different tissues were loaded to $10 \%$ Acr-Bis SDS-PAGE, and after transferring and blocking in 5\% milk, the membrane was probed with primary antibody against mCherry (1:200, BioVision, USA) or $\beta$-actin (1:10,000, Sigma-Aldrich, USA).

\section{4 mCherry image}

Mice were anesthetized by intraperitoneal injection of $1.25 \%$ avertin at a dose of $200 \mu \mathrm{L}$ per $10 \mathrm{~g}$ weight, followed by perfusion of phosphate buffered saline (PBS, pH 7.4) and $4 \%$ paraformaldehyde (PFA) in PBS. The whole brain was dissected and imaged with a fluorescence stereomicroscope (Leica MZ16F, Germany) equipped with a digital camera (Olympus DP71, Japan). mCherry signal was detected at DsRed2 channel. For sections analysis, the brain or eye was post-fixed in $4 \%$ PFA for $12-24 \mathrm{~h}$ at $4^{\circ} \mathrm{C}$, and equilibrated in $30 \%$ sucrose overnight at $4{ }^{\circ} \mathrm{C}$. Tissues were embedded in Tissue Freezing Medium (Leica, Germany), and $20-40 \mu \mathrm{m}$ sagittal sections were prepared in the cryostat (Leica). After rinsing in PBS for three times, the sections were directly observed under Olympus IX81 FV1000 confocal microscope (Olympus, Japan) using 559-nm laser. Images were acquired by capturing $1 \mu \mathrm{m}$-interval serial sec- 
tions, and z-stack projected for better performance.

\subsection{Neuronal and explant culture}

The detailed protocol for neuronal culture was adapted from elsewhere [9]. Briefly, the cerebellum and hippocampus were isolated from mouse at postnatal day 0.5 , and digested with TrypLETM (Life Technologies, USA) for $10 \mathrm{~min}$ at $37^{\circ} \mathrm{C}$, and went through a fire-polished glass pipette for a single cell suspension. Neurons $\left(4-5 \times 10^{4}\right)$ were plated on an $8 \mathrm{~mm} \times 8 \mathrm{~mm}$ coverslips precoated with $100 \mu \mathrm{g} \mathrm{mL}$ Poly-D-Lysine (Sigma-Aldrich) in Dulbecco's modified Eagle's medium with $10 \%$ fetal bovine serum for $4 \mathrm{~h}$. The medium was changed into Neuralbasal ${ }^{\mathrm{TM}}$ (Life Technologies) medium supplemented with 2\% B-27 (Life Technologies), $1 \times$ Glutamax $^{\mathrm{TM}}$ (Life Technologies), and $1 \times$ PenStrep (Life Technologies). Cells were cultured for further two days before fix for immunostaining. For the endogenous Class III $\beta$-tubulin visualization, neurons were fixed in $4 \%$ PFA for $30 \mathrm{~min}$ and permeabilized by $0.5 \%$ Triton X-100. After blocking in 5\% normal goat serum (Millipore, USA), cells were immunostained by primary antibody against Class III $\beta$-tubulin (TU-20, 1:100, Millipore), followed by a secondary antibody of FITC conjugated goat anti mouse IgG (1:200, Sigma-Aldrich). Cerebellar EGL explants were prepared from P3 mice and cultured on laminin $\left(10 \mu \mathrm{gL}^{-1}\right.$, Sigma-Aldrich) coated $8 \mathrm{~mm} \times 8 \mathrm{~mm}$ coverslips in Neuralbasal $^{\mathrm{TM}}$ (Life Technologies) medium supplemented with $2 \%$ B-27 (Life Technologies), 1× Glutamax ${ }^{\text {TM }}$ (Life Technologies), and $1 \times$ PenStrep (Life Technologies) for $48 \mathrm{~h}$. Explants were fixed in 4\% PFA in PBS and directly observed under Olympus IX81 FV1000 confocal microscope (Olympus) using 559-nm laser to detect the neurites.

\section{Results}

\subsection{Generation of Tubb3-mCherry BAC transgenic mouse line}

Using the modified BAC described in Materials and methods, we replace the stop codon of Tubb3 by mCherry complementary DNA (cDNA), as well as a linker sequence encoding Gly-Gly-Ser-Gly-Gly peptide, to make a transgenic mouse that expresses a carboxy-terminus tagged fusion Class III $\beta$-tubulin (Figure 1A). We fused mCherry to the carboxy-terminus of Class III $\beta$-tubulin because it was reported that the amino-terminus of each tubulin monomer located inside the molecule [10], and it was possible to lead to unsuccessful incorporation into microtubules of amino-terminus tagged fusion proteins. On the other hand, the two helices in the carboxy-terminus sit on the surface of the molecule [10] and a mouse $\beta$-tubulin fused to green fluorescent protein (GFP) at the carboxy-terminus was incorporated into microtubules and showed normal dynamics [2].
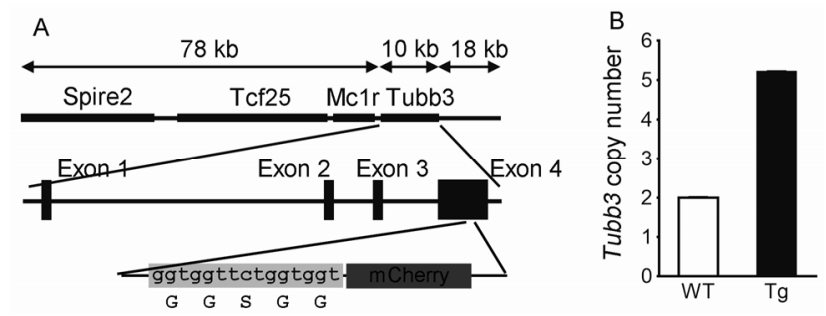

Figure 1 Generation of Tubb3-mCherry BAC transgenic mouse line. A, schematic representation of the modified BAC bMQ125_j13 containing the Tubb3 gene. The mCherry cDNA was inserted in frame $3^{\prime}$ of the coding region of $T u b b 3$ via homologous recombination. Between the coding region of Tubb3 and mCherry cDNA was a 15-bp sequence, which encodes a Gly-Gly-Ser-Gly-Gly linker. B, quantitative real-time analysis of wild-type and transgenic genomic DNA to determine transgene copy number from four repeated experiments.

We speculate that in our BAC transgenic mouse, mCherry fused Class III $\beta$-tubulin could be incorporated into neuronal microtubules without any interference to microtubule dynamics in the growing neurites. Initially, four transgenic founders were generated from injected fertilized eggs, and after a preliminary determination of transgene expression, the most robust expressing line was maintained and reported here. Using quantitative real-time PCR based method, we determined the Tubb3 gene copy number of this line. We found there were three Tubb3-mCherry copies integrated in the transgenic genome, as wild type genome has two endogenous copies of Tubb3 while the transgene has five in total (Figure 1B). Our transgenic mice were viable, fertile and had no phenotypical abnormality during over 18-month maintenance, implying that the transgenic expression of the fusing protein did not lead to cytotoxicity.

\subsection{Tubb3-mCherry fusion protein is expressed in various tissues in the adult transgenic mouse}

We first characterized the fusion protein expression in different tissues by Western blot. mCherry is a $\sim 30 \mathrm{kD}$ protein, but when it is fused with Class III $\beta$-tubulin, the mCherry antibody recognized a near $90 \mathrm{kD}$ protein, confirming the insertion of mCherry cDNA downstream of Tubb3 did not affect its expression (Figure 2A). As expected, the expression of the fusion protein was consistent with the endogenous protein, showing a strong expression in the nervous system and testes. The expression of the fusion protein in the testes may make our transgenic mice more useful beyond the nervous system, as the BAC based model is a better genetic tool to study the regulation of $T u b b 3$ expression in sertoli cells [11].

To further confirm the expression pattern of mCherry in the transgenic mouse, we observed strong mCherry fluorescence in whole brain and testes (Figure 2B and C). There was no phenotypical difference between wild type and transgenic animal in the brain or testes, supporting the mCherry incorporation did not interfere with their normal 
A
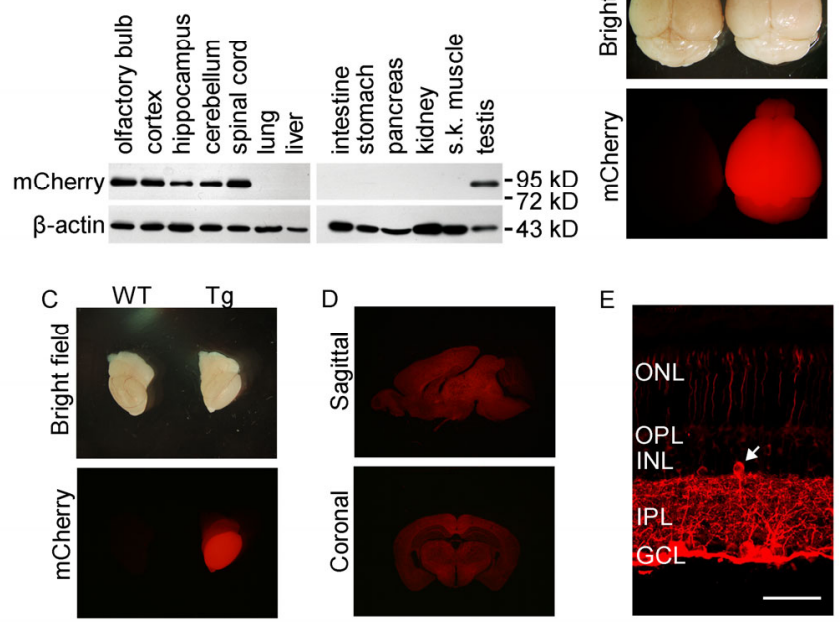

Figure 2 Tubb3-mCherry fusion protein is expressed in the adult transgenic mouse. A, Western blot analysis of protein lysates prepared from various tissues in adult transgenic mouse. mCherry antibody recognized the fusion protein (about $90 \mathrm{kD}$ ) expressed in different regions of the nervous system and the testes. B and C, Whole mount image of wild type (WT) and transgenic (Tg) animal brain (B) or testes (C). mCherry fluorescent signal was detected in transgenic animal but not in wild type control under the same density and exposure time. D, Sagittal and coronal brain section analysis to transgenic brain. mCherry fluorescent signal was detected in all the brain regions. E, mCherry fusion protein expression in the retina. Ganglion cells strongly expressed the fusion protein while some other cells in the retina including certain amacrine cells, photoreceptor cells, horizontal cells and bipolar cells expressed mCherry at a relatively low level. Scale bar, $50 \mu \mathrm{m}$. ONL, outer nuclear layer; OPL, outer plexiform layer; INL, inner nuclear layer; IPL, inner plexiform layer; GCL, ganglion cell layer. development. mCherry fluorescence analysis from sagittal and coronal brain sections suggested that the fusion protein was expressed in all adult brain regions, although mCherry fluorescence signal was stronger in the cerebral cortex than that in the cerebellum (Figure 2D). Besides the robust fusion protein expression in the brain, we also observed mCherry positive neurons in the retina. Retina ganglion cell layer (GCL) and inner plexiform layer (IPL) mainly consist of ganglion cell body and dendrites, respectively. Class III $\beta$-tubulin was often used as a ganglion cell marker and as expected, the expression of the fusion protein in GCL and IPL was relatively high (Figure 2E). It is noteworthy that the expression of the fusion protein was not solely located in the ganglion cells. Consistent with other report which used Tuj-1 antibody to determine Class III $\beta$-tubulin expression in the retina [12], we found some photoreceptor cells, certain amacrine cells (Figure 2E, arrow), some horizontal cells and bipolar cells also express the fusion protein, although the latter two showed a low fluorescent signal (Figure 2E).

\subsection{The expression of Tubb3-mCherry fusion overlaps with that of endogenous Class III $\beta$-tubulin}

We carried out a microexplant assay to identify whether our transgenic mouse can be used in cell culture system and label microtubules in developing neurons. Cerebellar external granule layer (EGL) explants were cultured in vitro for 2 $\mathrm{d}$, and fixed for mCherry imaging. We observed that mCherry signal was accumulated in the neurites (Figure 3A), supporting that our transgenic mouse could be used to study
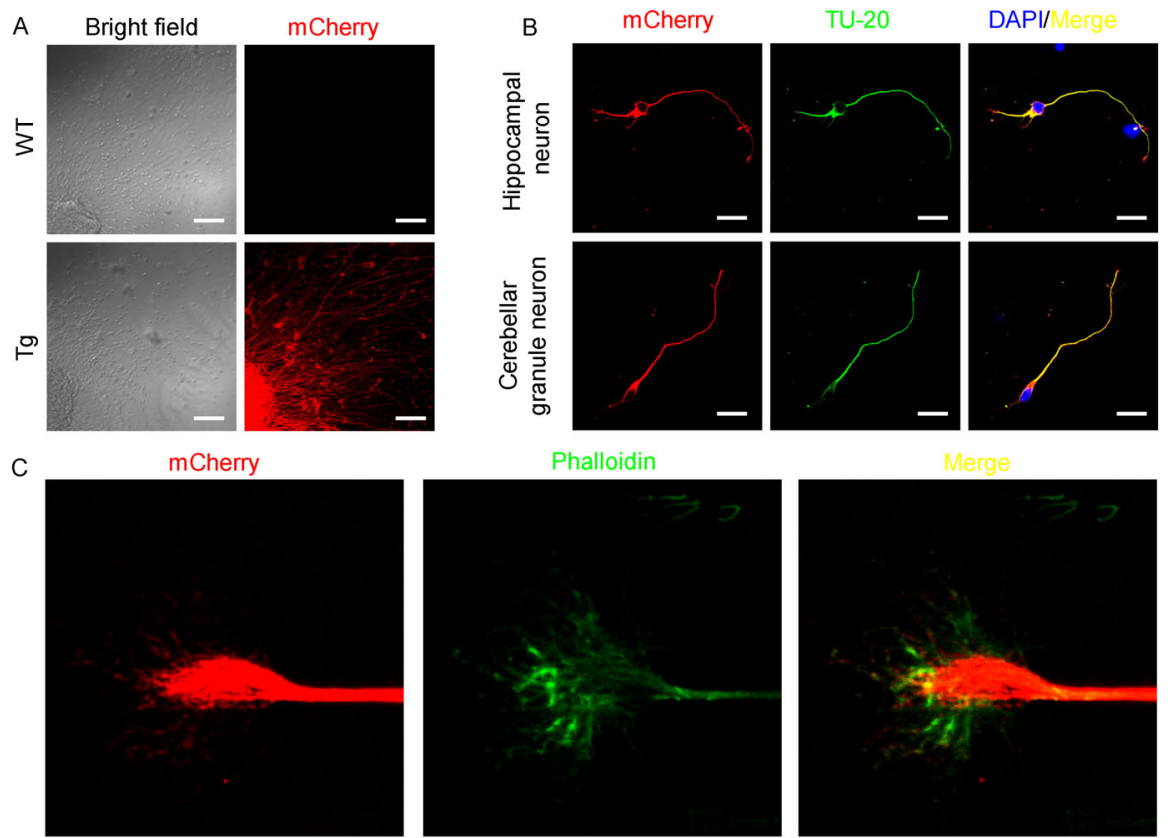

Figure 3 The expression of Tubb3-mCherry fusion protein overlaps with that of endogenous Class III $\beta$-tubulin. A, mCherry fluorescent signal was detected in the extending neurites of transgenic explant but not in the wild type neuritis. Cerebellar EGL explant from wild type and transgenic animal were cultured for $48 \mathrm{~h}$ and fixed for mCherry image under confocal microscope. Scale bar, $100 \mu \mathrm{m}$. B, mCherry fluorescence overlapped well with antibody associated signal in isolated hippocampal neurons (upper panel) and cerebellar granule neurons (lower panel). Both kinds of neurons were cultured for $48 \mathrm{~h}$ and immunostained with TU-20. Scale bar, $20 \mu \mathrm{m}$. 
the tangential migration and neurite outgrowth and guidance of cerebellar granule neurons. To assess whether the mCherry fluorescence could represent the neuronal microtubules, we also cultured isolated cerebellar granule neurons and hippocampal neurons, and immunostained with the endogenous Class III $\beta$-tubulin using an extensively used antibody TU-20. We found that mCherry signal of transgenic expression of the fusion protein overlapped with antibody-conjugated fluorescence in both the cultured neuronal types (Figure 3B). Although this experiment was carried out in fixed cells, there is no doubt that mCherry could also label microtubules in live neurons.

\subsection{Tubb3-mCherry fusion protein labels Purkinje cell dendrites during cerebellar development}

We here take the advantage of the transgenic animal to study postnatal cerebellar development. Previous study using adeno-associated virus (AAV) mediated gene transfer to infect the cerebellar Purkinje cells had demonstrated well about the Purkinje cell dendritic arborization behavior [13]. Compared to AAV method, although our transgenic fluorescence could not label a single neuron, visualization of all the neurons in the cerebellum may also deepen our knowledge on its development, and the genetic approach is surgery-free, which makes the labeling experiment more convenient. In the adult cerebellum at postnatal day 50 (Figure 4), the mCherry fluorescence could be clearly observed in the mature Purkinje cell dendrites, including both the primary and secondary branches. At postnatal day 10, dendrites of Purkinje cells could not be labeled clearly, con- sistent with the fact that Purkinje cell dendrites are not fully developed at this stage. Purkinje dendrites could be clearly visualized during the third postnatal week (P14 to P21), and the dendritic pattern became more and more complex. On the other hand, external granule layer illuminated by robust mCherry fluorescence gradually disappeared at the same stages. The strong mCherry fluorescence at the external granule layer may represent the process of developing granule neurons, which underwent tangential migration in the external granule layer. This example demonstrated that our Tubb3-mCherry transgenic mouse could be used for labeling neurons, especially Purkinje cell dendrites during cerebellar development.

\section{Discussion}

There has been a transgenic mouse Class-III $\beta$ tubulin reporter which expresses yellow fluorescent protein (YFP) under the control of a 3-kb promoter [14]. This transgenic mouse line could indeed report the Tubb3 expression during neuronal development, but there were some spatial and temporal differences between transgene expression and the endogenous Class III $\beta$-tubulin. The inconsistence was not strange because this was a conventional transgenic mouse line that the $3-\mathrm{kb}$ promoter used may not contain all the regulatory elements. It had been discussed that large foreign DNA fragment (such as a BAC) can be expressed independently of the integration site in transgenic animals $[15,16]$, we can overcome the problem easily by taking the advantage of BAC transgene, and hopefully the 107-kb
$\mathrm{P} 10$

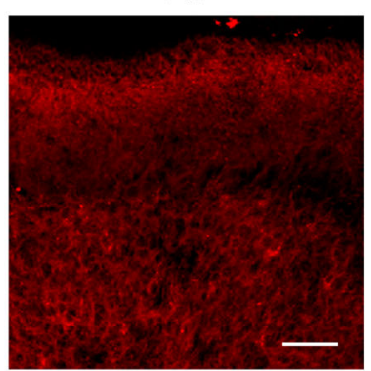

P18

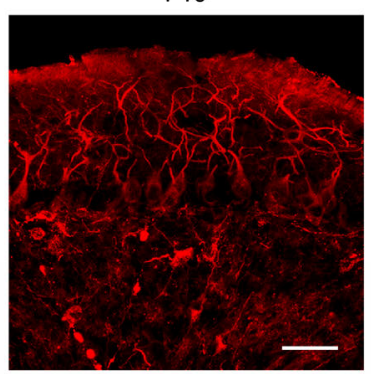

$\mathrm{P} 12$

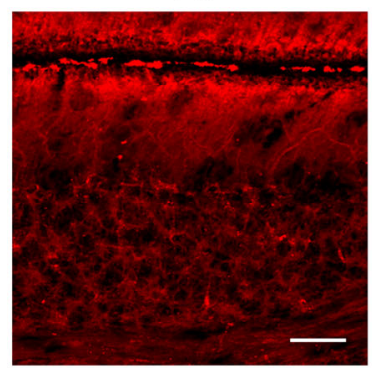

$\mathrm{P} 21$

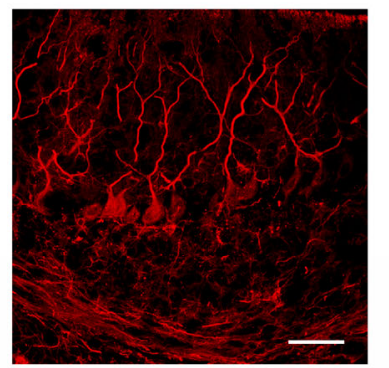

$\mathrm{P} 14$

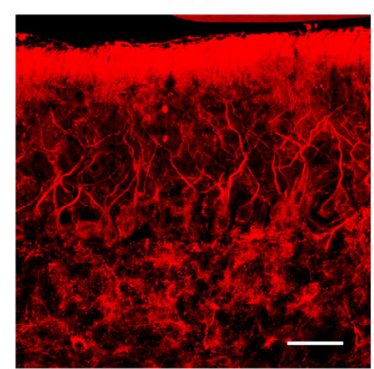

P50

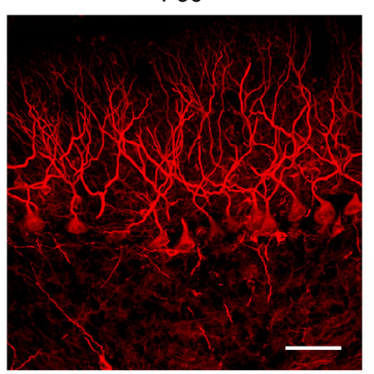

Figure 4 Tubb3-mCherry fusion protein labels Purkinje cell dendrites during cerebellar development. Strong mCherry fluorescence was observed after postnatal day 12 in the sagittal sections of transgenic cerebellum, and mainly distributed in the Purkinje cell dendrites located in the molecular layer. Scale bar, $50 \mu \mathrm{m}$. 
BAC may contain all the regulatory elements for Tubb3 expression without any positional effect. On the other hand, the BAC we used also carries genes other than Tubb3, including part of Spire2, Tcf 25 and Mclr. Integrating the BAC into transgenic mouse genome may lead to overexpression of these genes, which further interferes with normal cellular processes. However, our result showing a quite normal phenotype of the transgenic line may exclude this possibility.

Another benefit that our mouse has over the previous YFP reporter is the employing of mCherry, a newly developed red fluorescent protein. The use of mCherry to generate transgenic mouse and the advantage over other fluorescent proteins had been discussed elsewhere [17]. mCherry exhibits identical tolerance with either $\mathrm{N}$-terminal or C-terminal fusion versions, and mCherry- $\alpha$-tubulin fusion protein incorporated into microtubules successfully [18]. In addition, our mCherry transgenic mouse may be useful for colocalization assay with GFP.

The authors thank Prof. Han ShengCheng for offering the mCherry plasmid, and all lab members for discussions and suggestions.

1 Dent EW, Gupton SL, Gertler FB. The growth cone cytoskeleton in axon outgrowth and guidance. Cold Spring Harb Perspect Biol, 2011, 3: pii: a001800

2 Ludin B, Matus A. GFP illuminates the cytoskeleton. Trends Cell Biol, 1998, 8: 72-77

3 Stepanova T, Slemmer J, Hoogenraad CC, Lansbergen G, Dortland B, De Zeeuw CI, Grosveld F, van Cappellen G, Akhmanova A, Galjart $\mathrm{N}$. Visualization of microtubule growth in cultured neurons via the use of EB3-GFP (end-binding protein 3-green fluorescent protein). J Neurosci, 2003, 23: 2655-2664

4 Deinhardt K, Kim T, Spellman DS, Mains RE, Eipper BA, Neubert TA, Chao MV, Hempstead BL. Neuronal growth cone retraction relies on proneurotrophin receptor signaling through Rac. Sci Signal, 2011, 4: ra82

5 Riedl J, Flynn KC, Raducanu A, Gartner F, Beck G, Bosl M, Bradke F, Massberg S, Aszodi A, Sixt M, Wedlich-Soldner R. Lifeact mice for studying F-actin dynamics. Nat Meth, 2010, 7: 168-169
6 Sullivan KF. Structure and utilization of tubulin isotypes. Ann Rev Cell Biol, 1988, 4: 687-716

7 Lee MK, Rebhun LI, Frankfurter A. Posttranslational modification of class III beta-tubulin. Proc Natl Acad Sci USA, 1990, 87: 7195-7199

8 Tischfield MA, Baris HN, Wu C, Rudolph G, Van Maldergem L, He W, Chan WM, Andrews C, Demer JL, Robertson RL, Mackey DA, Ruddle JB, Bird TD, Gottlob I, Pieh C, Traboulsi EI, Pomeroy SL, Hunter DG, Soul JS, Newlin A, Sabol LJ, Doherty EJ, de Uzcátegui CE, de Uzcátegui N, Collins MLZ, Sener EC, Wabbels B, Hellebrand $\mathrm{H}$, Meitinger $\mathrm{T}$, de Berardinis $\mathrm{T}$, Magli A, Schiavi C, Pastore-Trossello M, Koc F, Wong AM, Levin AV, Geraghty MT, Descartes M, Flaherty M, Jamieson RV, Møller HU, Meuthen I, Callen DF, Kerwin J, Lindsay S, Meindl A, Gupta Jr ML, Pellman D, Engle EC. Human tubb3 mutations perturb microtubule dynamics, kinesin interactions, and axon guidance. Cell, 2010, 140: 74-87

9 Peng YJ, He WQ, Tang J, Tao T, Chen C, Gao YQ, Zhang WC, He XY, Dai YY, Zhu NC, Lv N, Zhang CH, Qiao YN, Zhao LP, Gao X, Zhu MS. Trio is a key guanine nucleotide exchange factor coordinating regulation of the migration and morphogenesis of granule cells in the developing cerebellum. J Biol Chem, 2010, 285: 24834-24844

10 Nogales E, Wolf SG, Downing KH. Structure of the $\alpha \beta$ tubulin dimer by electron crystallography. Nature, 1998, 391: 199-203

11 De Gendt K, Denolet E, Willems A, Daniels VW, Clinckemalie L, Denayer S, Wilkinson MF, Claessens F, Swinnen JV, Verhoeven G. Expression of Tubb3, a beta-tubulin isotype, is regulated by androgens in mouse and rat sertoli cells. Biol Reprod, 2011, 85: 934-945

12 Sharma RK, Netland PA. Early born lineage of retinal neurons express class III $\beta$-tubulin isotype. Brain Res, 2007, 1176: 11-17

13 Chédotal A, Kaneko M, Yamaguchi K, Eiraku M, Sato M, Takata N, Kiyohara Y, Mishina M, Hirase H, Hashikawa T, Kengaku M. Remodeling of monoplanar purkinje cell dendrites during cerebellar circuit formation. PLoS One, 2011, 6: e20108

14 Liu L, Geisert EE, Frankfurter A, Spano AJ, Jiang CX, Yue J, Dragatsis I, Goldowitz D. A transgenic mouse class-III beta tubulin reporter using yellow fluorescent protein. Genesis, 2007, 45: 560-569

15 Giraldo P, Montoliu L. Size matters: use of YACs, BACs and PACs in transgenic animals. Transgenic Res, 2001, 10: 83-103

16 Heintz N. Bac to the future: the use of BAC transgenic mice for neuroscience research. Nat Rev Neurosci, 2001, 2: 861-870

17 Fink D, Wohrer S, Pfeffer M, Tombe T, Ong CJ, Sorensen PHB. Ubiquitous expression of the monomeric red fluorescent protein mCherry in transgenic mice. Genesis, 2010, 48: 723-729

18 Shaner NC, Campbell RE, Steinbach PA, Giepmans BNG, Palmer $\mathrm{AE}$, Tsien RY. Improved monomeric red, orange and yellow fluorescent proteins derived from Discosoma sp. red fluorescent protein. Nat Biotech, 2004, 22: 1567-1572

Open Access This article is distributed under the terms of the Creative Commons Attribution License which permits any use, distribution, and reproduction in any medium, provided the original author(s) and source are credited. 